\title{
Genre Analysis of Low-rated and High-rated Literature Reviews
}

\section{by EFL Postgraduates}

\author{
Cheng-hua Hsiao ${ }^{1}$ \\ ${ }^{1}$ Department of Foreign Languages and Literature, National Ilan University, Ilan City, Tawan \\ Correspondence: Cheng-hua Hsiao, Department of Foreign Languages and Literature, National Ilan University. \\ Address: No. 1, Shengnong Rd. Sec. 1, Yilan City, Yilan County, Taiwan. (26047). Tel: 886-3-935-7400. E-mail: \\ chhsiao@niu.edu.tw
}

Received: July 5, 2015 Accepted: July 20, 2015 Online Published: October 12, 2015

doi:10.5430/ijelt.v3n1p1 URL: http://dx.doi.org/10.5430/ijelt.v3n1p1

\begin{abstract}
Literature review (hereafter LR) discourse had not been a recognized genre until its move structure was formulated (Kwan, 2006; Hsiao \& Yu, 2008). However, postgraduates keep raising difficulties for this particular genre (Belcher, 1994; Dong, 1998; Qian, 2003; Liu, 2007; Kwan, 2008). Therefore, the move combinations in terms of "move configuration" was studied, by which it was a textual unit that could be readily recognized using sections with aims to analyze literature review quality levels and to provide a pedagogical implication for thesis instructors. In this study, Hsiao and Yu's LR framework (2012) were adopted and thirty LR discourses were collected and rated into two cohorts- low-rated and high-rated groups. The results revealed that the rhetorical strategies reflected in high-rated literature reviews have complicated move configurations and more move cycles than low-rated ones. Listing and author prominent citations are found to be the text features for the low-rated LRs whereas information prominent citation is the key text feature to summarize the contents from multiple source articles in high-rated ones.
\end{abstract}

Keywords: literature review, genre analysis, English for Academic Purposes (EAP)

\section{Introduction}

Literature review discourse is a particular genre that postgraduates must familiarize themselves with during their course of study. Past literature show that substantial difficulties exist in literature review writing and how segments of texts (moves) are arranged in literature review discourse composed by post-graduate writers remains unclear. In this study, an attempt was made to examine how the moves of this particular genre are combined in sections of this particular genre to promote exploitation with pedagogical implications.

Moves studies originated from Swales' genre-based theory (1990), which has been central to English for Specific Purposes (hereafter ESP). ESP sees genre both as a text structure and adopts a view that genre is a "staged and goal-oriented social process" (Martin, 1992, p. 5), which has been inspired by Hallidayan conception of linguistic and textual realization of social purposes (Halliday, 1994; Halliday \& Hasan, 1989; Martin, 1992). Text conventions are regarded as organizing messages so readers can promptly recognize the intended messages. ESP researchers tend to relate texts to more specific communities that use genres to promote their goals, such as academic disciplines or particular professions.

Swales (1990), influenced by the focus upon function and purpose in discourse, investigated the rhetorical moves. His research, focusing on how specific rhetorical structures used in a specific text or genre, resulted in discovering the relationship among form, function and genre. His canonical work, genre analysis, particularly the move analysis for the introduction in research paper, has inspired many subsequent researches in this field. The most well-known move analysis is Swales' CARS Model. CARS is a term for "Creating-A-Research-Space" in the introductions of research articles. Swales (1990) formulated a move structure for RA introductions in order to offer a generic view on how RA introductions are textually constructed. In this move structure, RA introductions usually start with a move that establishes thematic areas of study. RA authors, subsequently, create a niche using a number of options as indicated in steps. Last, authors occupy the niche by reporting their particular concerns for proposed studies. This 
line of studies is more applied because it has been motivated by a pedagogical concern with language teaching (Bhatia, 1993). The accumulated research has shown that specific genre can be identified on the basis of purposes or communicative goals that the texts set out to achieve.

Move, a functional term, is a basic unit in conducting genre analysis and many scholars (Lores, 2004; Nwogu, 1997; Santo, 1996; Swales, 1990; Swales \& Feak, 2000) have each contributed to the definition of move. Move analysis have been conducted following Swales's study; many researchers devoted to examine other research genres in RA, including abstract (Bhatia, 1993; Lau, 2003; Lores, 2004; Santo, 1996), methods (Nwogu, 1997; Lim, 2006), results (Brett, 1994; Posteguillo, 1999; Yang \& Allison 2003; Williams, 1999), discussion (Dudley-Evans 1986; Hopkins and Dudley-Evans, 1988), conclusion (Bunton, 2005; Hewings, 1993; Yang \& Allison, 2003) and acknowledgement (Hyland, 2004).

Kwan's model (2006) draws insights from Swales' $(1981,1990)$ genre-analysis model and attempted to examine the move structure of literature review by adapting part of Swales' CARS model and to identify the move structure of LRs among 20 doctoral dissertations in applied linguistics. Hsiao and $\mathrm{Yu}$ (2012) described move use more specifically to derive operational definitions for the moves identified from student literature review writing. They identified the following four major moves from literature review chapters in graduate theses on applied linguistics by Taiwanese students: (1) conceptualizing themes in a field, (2) integrating previous studies on the themes, (3) relating previous studies to the current study, and (4) advancing personal research.

However, Kwan (2006) and Hsiao and Yu (2012) have considered only the move identification in literature chapters in theses and dissertations; however, how moves are developed and combined within themes has been under-researched. How are the moves described in the move structure combined and presented in theme-based literature reviews written by student writers and how student writers use the moves to achieve their communicative purposes in literature review discourse remain vague. Are the moves presented in a linear or cyclic manner? Few studies have explored the sophisticated schematic structure and established an evaluative scale on literature review writing.

With the assumption that thesis writers at different levels use particular move combinations to achieve their respective communicative purposes, we described target second language (L2) writers' move distribution and move configurations. By move distribution, I mean the frequency of moves use in the literature review move structure while, by move configuration, I mean the unit of combinations of moves in sections. By calculating and identifying the moves frequency and move configurations can these writers' rhetorical strategies in composing their literature review be revealed. Additionally, to derive the literature review texts, we divided the data into two cohorts, namely low-rated and high-rated groups, to identify the levels of quality reflected in rhetorical moves and constituent moves.

To investigate the move arrangements of literature reviews in MA theses written by Taiwanese graduates studying teaching English as a foreign language (TEFL), this study raised the following research questions:

1) What is the move distribution of literature review discourse at low-rated and high-rated levels?

2) What are the move configurations at these two levels?

3) What are the text features at each level?

\section{Literature Review}

Studies on literature review discourse are reviewed, including literature review (hereafter LR) move structures, criteria of quality literature review, nonnative student writers' literature review writing process and the difficulties writing up literature reviews. Evaluation of LR helps us to the norms for qualities of literature review discourse while reviewing nonnative speakers' writing process and difficulties in writing up literature reviews assist us perceive LR writing inadequacies that they have had.

\subsection{Two Move Structures of Literature Reviews}

Despite the little attention paid to literature reviews, Kwan (2006) attempted to adapt part of the CARS model and identify the move structure of literature reviews. In the result framework, three moves were identified: (1) establishing a thematic territory, (2) evaluating the state of the field, and (3) announcing personal research. By contrast, Hsiao and $\mathrm{Yu}$ (2012) described move use more specifically, deriving operational definitions for literature review moves from students' literature review writing. Hsiao and Yu's study (2012) denoted four levels of moves: conceptualizing themes in a field (Move 1), integrating previous works on the themes (Move 2), relating the previous works to the current study (Move 3), and advancing to personal research (Move 4). In this study, Hsiao and Yu's 
framework was adopted based on the two main reasons. First, the move uses described their study are more specifically to derive from MA student literature review writings. Second, the ambiguity and confusion in "claiming the centrality of the themes reviewed (1B)" and "making confirmative claims (2A)," two submoves in Kwan's move framework, may be avoided. In addition, some of the sequence of moves are restructured in Hsiao and Yu's move structure from locally to globally.

\subsection{Criteria of Quality Literature Reviews}

Some criteria have been presented to establish the evaluation of literature reviews (Boote \& Beile, 2005; Granello, 2001; Moravcsik \& Murugesan, 1975) because Boote and Beile (2005) outlined five areas for evaluation: coverage, synthesis, methodology, significance, and rhetoric. Granello (2001) focused on analyzing the cognitive levels that literature reviews present. The cognitive hierarchy is divided into six levels: (1) knowledge, (2) comprehension, (3) application, (4) analysis, (5) synthesis, and (6) evaluation. In the six hierarchical orders of cognition, Granello considered the taxonomy by Bloom (1956) on the cognitive domains of educational activities (Granello, 2001, pp. 296-297). Moravesik and Murugesan (1975) discussed the characteristics of literature reviews, which are categorized into four types: conceptual and operational, evolutionary and juxtapositional, organic and juxtapositional, and conformational and negational.

In addition, some authors have acknowledged the importance of literature review and established criteria for quality literature reviews (Lather, 1999; Strike \& Posner, 1983). Lather (1999) maintained that a critical role should provide a synthetic review to produce a new productive work, rather than merely mirroring research in a field. Strike and Posner (1983, pp. 356-357) further suggested that three elements constitute a quality synthetic review: presenting the problems encountered in a field, clarifying and resolving the problems, and offering a new perspective with increased explanatory and predictive power. Based on these criteria, the communicative moves involved in move arrangement must incorporate the aforementioned elements in the rhetorical agenda of a literature review.

\subsection{Nonnative Postgraduates' Literature Review Writing Process}

Many scholars have devoted their research to examining and constructing literature review writing for nonnative speakers of English (Belcher, 1994; Dong, 1998; Qian, 2003; Liu, 2007; Kwan, 2008). Qian (2003) presented four case studies on how Chinese people addressed literature review writing in Canada. He attributed the struggles of these students in literature review writing to limited vocabulary, sentence-level difficulties, and paraphrasing difficulties. The strategies they used to compensate for their lack of competence were reading and modeling, planning, using first language and translation, and communicating with supervisors. Liu (2007) observed the literature review writing process of a Taiwanese graduate student in applied linguistics. In this case study, Liu found that the writer could hardly find the research niche. Move use tended to be unsophisticated when examined using Kwan's move structure. Kwan (2008) upheld "the demarcation view of the processes of reading, writing and researching" (p. 42). She contended that these advanced literacy activities are mutually related. Kwan found that doctoral students at various stages of learning literature review discourse adopted different strategies. Reading at the beginning stage seemed to be the least focused. As reading developed, various themes related to conceptualizations, methods, and theories were interconnected to gradually compose a complete literature review. At the later stage, the students were intensively engaged in various research activities. The literature the students produced during this period aided them in identifying gaps that required reading for specific details. Reading for the literature review at the final stage enabled updating information and citing works they read. However, these studies have not provided a clear idea of the textual construction in the literature review discourse written by these writers.

The sections of literature review have discussed various aspects of nonnative student writers' LR writing. However, the gap so far lies in how the moves are combined and configured in LR writing, which still remains unexplored. Therefore, this study aimed to find the move distribution and move configurations in literature review. By move distribution, it is defined as frequencies of move that LR writers use while by configuration, it refers to the unit of combination of moves in sections.

\section{Methodology}

\subsection{Data Collection}

Sampling for this study had four criteria: topics, regions, institutions, and year coverage. To ensure external validity, 30 theses were selected using stratified random sampling to ensure the findings of the study would be relevant not only to the research population but also to a wider population of language learners (Mackey \& Gass, 2005). In addition, the electronic files of the theses were obtained from libraries or relevant departments at universities with 
the permission of writers.

\subsection{Data Analysis}

For analysis, frequencies of major moves and submoves in the move structure were shown. Subsequently, move configuration and move cycle were analyzed. Next, the decision on the selection of raters, the rating scale, rating procedures and inter-rater reliability were specified. In addition, coding as a data analysis procedure is explained, in which the selection of coders and reliability issues were introduced.

\subsubsection{Distribution of Moves}

The researcher used frequency, percentage and mean to derive the move occurrence, and number of each submove used in an LR in the study. The frequency, percentage and mean of major moves and submoves were calculated. The LRs were divided into two major groups- the low-rated and the high-rated LRs, by which they were further grouped into two subgroups- the deficient and the poor groups for the low-rated level, the competent and the distinctive group for the high-rated level. All the submoves in these two major groups or four levels of LRs were also computed by their frequencies, percentages and means to look for the group patterns.

\subsubsection{Move Progression}

Move progression, as an analysis for this study, was to identify a series of moves that LR writers chose for their LRs. The researcher listed the move progressions of the four groups of LRs to examine if there was a regularity of move sequencing of LR in the writers' choice of moves.

\subsubsection{Move Configuration}

"Move configurations" refers to the unit of combination of moves in sections. The means of move configurations were calculated in this study. The researcher attempted to figure out the preference of the move configuration that the writers of each group use. The units of move configurations included "single move configuration," "2- move configuration," "3-move configuration," and "4-move configuration."

\subsubsection{Move Cycle}

Move cycle is understood as a recursive pattern from Move 1 to Move 4. Generally an ideal move cycle is identified as the sequence from Move 1 to Move 4. Move cycle is used to determine move appearances so as to determine the LR qualities.

\subsubsection{Rating}

Two procedures in the methods were introduced, which were considered important in assessing the reliability of this study - rating and coding. Rating in this study was to determine the quality of collected LRs because the pedagogical implication could be derived by distinguishing the various qualities of LRs. The procedures of rating involved with the rating scale, selection of raters, raters' training session, and formal rating procedures, which are to be presented in the following subsections.

\subsubsection{Rating Scales}

A rating scale was designed for this study to determine the quality of LRs; therefore, it was produced for selected raters to rate the collected LRs. The selected LRs in master's theses were categorized into four groups using the Rating Scale of MA Literature Reviews (See Appendix), which was developed by the researcher based on the suggestions of Boote \& Beile (2004), Granello (2001), and Hart (1999, p17).

For the rating scale, a holistic scoring rather than an analytic scoring was used in this study. Holistic scoring refers to the assignment of a single score to a piece of writing on the overall impression of it whereas analytic scoring involves the assignment of respective scores for different aspects of a writing task. This decision took Hugh's (2003) suggestion in that the concentration on several aspects may divert attention from the overall effect of the writing task. Analytic scoring may be reliable but may not valid. In addition, the aspects that are scored separately may not accurately represent the complete set of such aspects.

In the rating scale, there were four levels that explicated the quality of LR in master theses: Code 1 stood for "Deficient," Code 2 "Poor," Code 3 "Competent," and Code 4 "Distinctive." LRs marked Code 1 and Code 2 were attributed to low-rated LRs. LRs marked competent (Code 3) and distinctive (Code 4) were characterized as high-rated LRs, which generally have presented discussions of the results from the source articles and LRs were organized around themes or topics. 


\subsubsection{Selection of Raters}

Two raters were recruited to determine the qualities of LRs and to ensure reliability of this rating result. The two raters were two professors who were teaching academic writing in TEFL programs in Taiwan. Also, they had been conducting research in the TEFL field for the past five years when they were rating the collected LRs. In order to reach high inter-rater reliability, a training session for raters was also carried out. These qualifications were to ensure that they fully understood the contents of LRs and had sufficient field knowledge and genre knowledge to determine the qualities of LRs.

Inter-rater reliability was subsequently calculated using Cohen Kappa coefficient $(\kappa)$, which is generally thought to be a more robust measure than simple percent agreement calculation since $\kappa$ takes into account the agreement occurring by chance (Mackey \& Gass, 2005). This method was used because it perfectly worked for a situation where two raters were involved: it treated data as nominal and assumed that the ratings had no natural ordering. The inter-rater reliability derived was $73.33 \%$, which was considered substantial agreement.

\subsubsection{Coding}

Coding is especially important because all the results and analysis were based on coding. As indicated by many researchers (Crooks, 1986; Lores, 2004), lacking uniform standards in move identification is the major flaw of genre analysis and causes a validation problem. Move analysis tends to depend largely on coders' personal judgment, which may be quite difficult to replicate. In move analysis, the judgment for textual boundaries is content-based and is not linguistically constrained (Martin, 1992; Paltridge,1994; Swales, 1981). Therefore, the establishment of high reliability toward the analysis of text structure is important, as suggested by Crooks (1986). After the data were selected, I followed the steps suggested by Crooks in coding the moves: selection of coders, and calculation of inter-coder reliability.

\subsubsection{Selection of Coders}

Two coders were selected in order to reach the reliability of coding results. In order to reach high reliability, their educational backgrounds were demanded. In this study, the two coders, holding masters' degrees in TEFL, were currently $\mathrm{PhD}$ students in two respective TEFL programs. They were required to attend the coding training sessions prior to formal coding.

After the coding sessions were completed, the two coders read the literature reviews of the thirty master's theses. The textual boundaries of the thirty master theses were coded according to the definition of each move. They decided which submoves should be assigned to the corresponding texts and found the equivalent codes, marking them down on their collections.

\subsubsection{Inter-coder Reliability}

Inter-coder reliability is the degree of agreement between coders. It gives a score of how much homogeneity there is in the ratings given by judges. In this study, like the calculation of inter-rater reliability, Cohen Kappa coefficient $(\kappa)$ (Scott, 1955; Sim \& Wright, 2005) was used to measure categorical items. However, the discrepancy went through discussion until a substantial agreement was reached. The result of Cohen Kappan coefficient was 85.8\% for inter-coder reliability, which was considered substantial agreement.

\subsection{LR Move Structure}

The move structure by Hsiao and $\mathrm{Yu}$ (2012) comprising four major moves was adopted in this study to code the data. This framework was used because it was developed for literature reviews of graduate theses in applied linguistics and elucidated specific operational definitions of submoves. Table 1 presents the four major moves and their submoves.

Table 1. Move Structure of Literature Review
Move 1 Conceptualizing themes in a field
1A: Presenting field knowledge, theories, beliefs, or practices related to the current study
1B: Creating a historical context
$1 \mathrm{C}$ : Identifying subfields in the major field
1D: Organizing major theme(s) to be reviewed for the current study 
1E: Surveying research activities

\section{Move 2 Integrating previous studies on the themes}

2A: Grouping and drawing ideas from source materials to gain a new perspective

(ideas from various sources that support a theme are compiled and compared)

2B: Critiquing the quality of the research discussed

(weaknesses, strengths, and limitations, or a misplaced concept underlying the research, or unresolved conflicts among the authors of previous studies concerning a research topic)

\section{Move 3 Relating previous studies to the current study}

3A: Claiming relevance to the current study

3B: Indicating a gap

(a variable, factor, or research area that has been overlooked in past studies)

\section{Move 4 Advancing to personal research}

4A: Stating the aim of the research

4B: Stating the research design or research process

4C: Stating the theoretical position or theoretical framework

\section{Results and Discussion}

\subsection{Move Distribution}

Based on the rating results that 18 literature reviews were low-rated and 12 high-rated, we began to examine the differences of move use by writers at different levels.

Table 2. Frequency and Percentages of the High-rated and Low-rated Literature Reviews

\begin{tabular}{|c|c|c|c|c|c|c|}
\hline & \multicolumn{3}{|c|}{ Low-rated $(\mathrm{N}=18)$} & \multicolumn{3}{|c|}{ High-rated $(\mathrm{N}=12)$} \\
\hline & $\mathrm{F}^{*}$ & $\%$ & Mean & $\mathrm{F}$ & $\%$ & Mean \\
\hline $1 \mathrm{~A}$ & 95 & $41.48 \%$ & 5.28 & 59 & $25.54 \%$ & 4.92 \\
\hline 1B & 3 & $1.31 \%$ & 0.17 & 5 & $2.16 \%$ & 0.42 \\
\hline $1 \mathrm{C}$ & 1 & $0.44 \%$ & 0.06 & 4 & $1.73 \%$ & 0.33 \\
\hline $1 \mathrm{D}$ & 29 & $12.66 \%$ & 1.61 & 23 & $9.96 \%$ & 1.92 \\
\hline $1 \mathrm{E}$ & 30 & $13.10 \%$ & 1.67 & 13 & $5.63 \%$ & 1.08 \\
\hline $2 \mathrm{~A}$ & 35 & $15.28 \%$ & 1.94 & 49 & $21.21 \%$ & 4.08 \\
\hline $2 \mathrm{~B}$ & 6 & $2.62 \%$ & 0.33 & 13 & $5.63 \%$ & 1.08 \\
\hline $3 \mathrm{~A}$ & 12 & $5.24 \%$ & 0.67 & 8 & $3.46 \%$ & 0.67 \\
\hline 3B & 5 & $2.18 \%$ & 0.28 & 29 & $12.55 \%$ & 2.42 \\
\hline $4 \mathrm{~A}$ & 8 & $3.49 \%$ & 0.44 & 20 & $8.66 \%$ & 1.67 \\
\hline 4B & 5 & $2.18 \%$ & 0.28 & 6 & $2.60 \%$ & 0.50 \\
\hline $4 \mathrm{C}$ & 0 & $0.00 \%$ & 0.00 & 2 & $0.87 \%$ & 0.17 \\
\hline Total & 229 & & & 231 & & \\
\hline
\end{tabular}

*Frequency

One major pattern identified in Table 2 is that the means of move uses increases from Move 1 to Move 4 as the level advances. In other words, Move 3 and Move 4 are used more frequently by high-rated writers than by low-rated writers. Low-rated writers use $1 \mathrm{~A}$ and $1 \mathrm{E}$ most often while high-rated frequently use $2 \mathrm{~A}, 3 \mathrm{~B}$ and $4 \mathrm{~A}$. 
The aforementioned findings clearly reveal the qualities of the two groups of literature reviews. The major flaw in low literature reviews is that the move selection focused on presenting existing knowledge $(1 \mathrm{~A})$; few topical integrations or arguments of past literature $(2 \mathrm{~A})$ were found and no new information in the proposed studies was revealed. An occurrence of all four major moves was identified as the threshold of the high-rated group. In addition, the use of the four major moves was more balanced for high-rated group.

\subsection{Move Progression}

Move progression, as an analysis for this study, was to identify a series of moves that LR writers chose for their LRs. The researcher listed the move progressions of the four groups of LRs to examine if there was a regularity of move sequencing of LR in the writers' choice of moves.

Table 3. Move Progression of the Low-rated and High-rated LRs

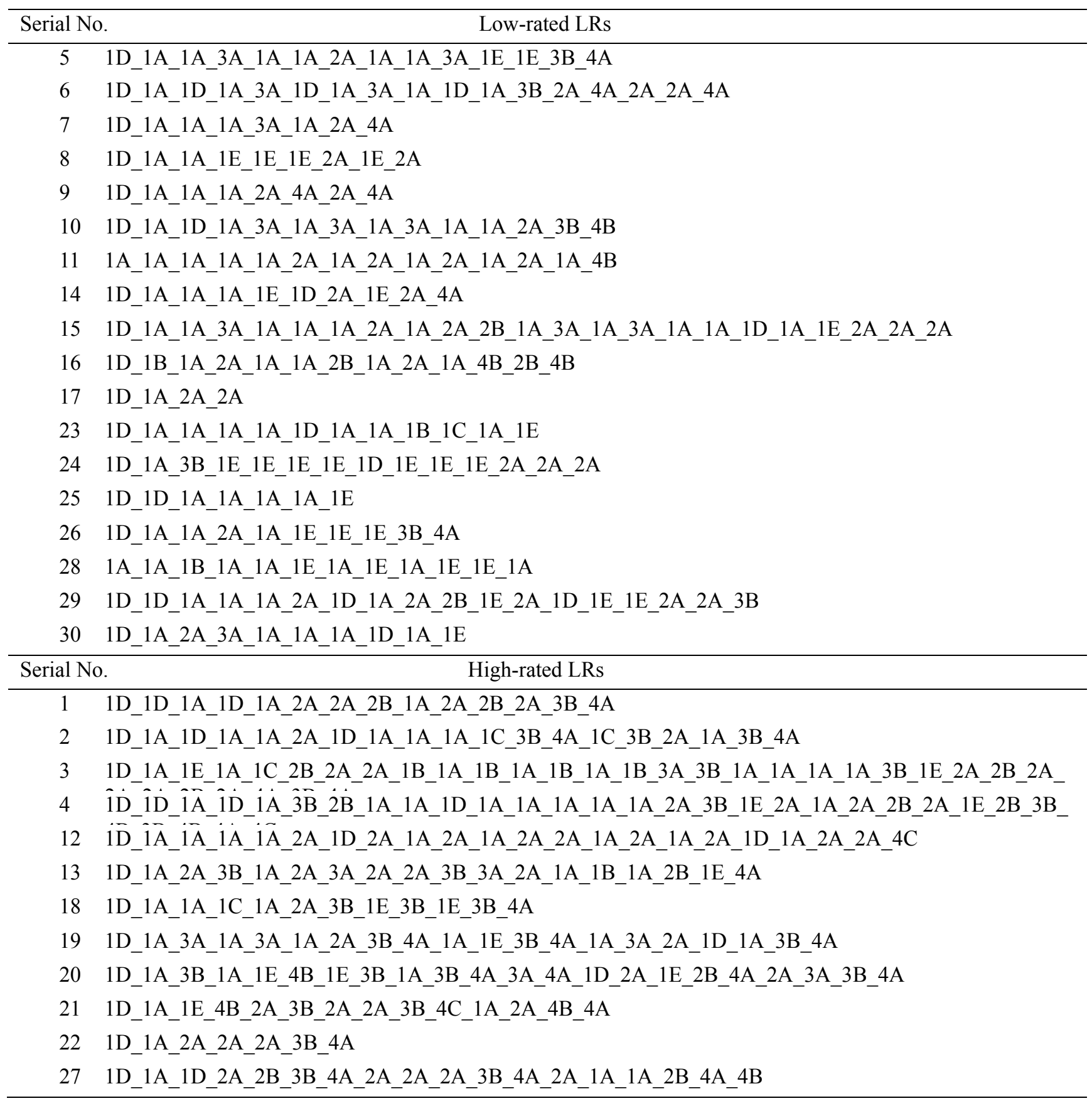




\subsection{Move Configuration}

To further investigate the move use, the moves were categorized on a section basis. The term "move configuration" represented the module of moves segregated by a section. Move configuration was the first textual unit that can be readily recognized using sections. A section was a large and visible unit that determined the textual boundary of a thematic discourse (Table 3). A configuration was used to describe move combinations and the complexity of a move structure. Move configurations were classified into four types to examine the patterns in move configuration preferred by writers at various levels: (1) single-move configuration, (2) two-move configuration, (3) three-move configuration, and (4) four-move configuration. "Single-move configuration" indicates the single or repeated use of submoves under one major move. "Two-move configuration" is any combination of submoves under two major moves. "Three-move configuration" includes any combination of submoves under three major moves. Move arrangements involving submoves under four major moves are "four-move configurations." Table 4 shows the relationship between the move structures in configurations and the literature review qualities.

Table 4. Average of Move Configuration Used in Two Groups

\begin{tabular}{lrlcl}
\hline LR quality & Low-rated & \multicolumn{3}{c}{ High-rated } \\
\hline Configuration Type & $\mathrm{N}=18$ & Average & $\mathrm{N}=12$ & Average \\
\hline Single move configuration & 104 & 5.78 & 26 & 2.17 \\
2-move configuration & 30 & 1.86 & 20 & 4.33 \\
3-move configuration & 5 & 0.30 & 18 & 1.50 \\
4- move configuration & 0 & 0.00 & 5 & 0.42 \\
\hline
\end{tabular}

Table 4 reveals that the high-rated literature reviews display more complex move configurations. As the level advances, complex configuration types increase. In the low-rated group, the single-move configuration was most frequently used, and the two-move configuration comprised the second largest proportion of move configuration use. In spite of the frequent use of single-move configurations, the 2-move and 3-move configurations were used more than once per literature review in the high-rated group.

\subsection{Qualities of Literature Review Discourses}

\subsubsection{The Low-rated Group}

Table 5 shows the move use by the low-rated writers. The number of the first row signifies the sections for each LR and the number in the first column indicates the serial number given to the LR. The symbol (composed of a number and a letter) in each cell indicates the move used in each section of the designated LR.

Table 5 showed that no progress on established theoretical grounds is made in reviewing studies on a topic because the initiated topics begin and halt at theme conceptualization (Move 1) in each section of the LRs in this deficient group.

Table 5. Sectional Move Use in the Low-rated Group

\begin{tabular}{|c|c|c|c|c|c|c|c|}
\hline Section & 1 & 2 & 3 & 4 & 5 & 6 & 7 \\
\hline 25 & $1 \mathrm{D}$ & $1 \mathrm{D}$ & $1 \mathrm{~A}$ & $1 \mathrm{~A}$ & $1 \mathrm{~A}$ & $1 \mathrm{~A}$ & $1 \mathrm{E}$ \\
\hline 28 & $1 \mathrm{~A}$ & $1 \mathrm{~A}$ & $1 \mathrm{~B}$ & $1 \mathrm{~A} \quad 1 \mathrm{~A} \quad 1 \mathrm{E} \quad 1 \mathrm{~A} \quad 1 \mathrm{E} 1 \mathrm{~A}$ & $1 \mathrm{E} 1 \mathrm{E}$ & $1 \mathrm{~A}$ & \\
\hline
\end{tabular}

No topic expansion or integration is identified in these two deficient LRs because the move use in the two deficient LRs all falls under Move 1 in all sections. The integration of the past studies (Move 2), the relevance to the current studies (Move 3) and the announcement of the current study (Move 4) are not found in this group. To facilitate the analysis of the move configurations, Table 6 is made to show the use of move configurations, which is classified by the four types of move configurations- "single move configuration," "2-move configuration," "3-move configuration," and "4-move configuration." These four configurations display the complexity of the move structure by the 
designated group. Starting moves (1D) are excluded in the frequency of the move configurations.

Table 6. Frequency of Move Configurations in Sections in Deficient Group

\begin{tabular}{ll}
\hline Move Configurations & Frequency \\
\hline Single move configuration & 6 \\
1A & 1 \\
1B & 1 \\
1E & 1 \\
1D & 1 \\
1A_1A_1E_1A_1E_1A & 1 \\
1E_1E & \\
2-move configuration & 0 \\
$\quad$ N/A & \\
3-move configuration & 0 \\
$\quad$ N/A & \\
4-move configuration & 0 \\
\hline Total & 11 \\
\hline
\end{tabular}

Single move configuration is found to be the dominant move configuration in this group and no topic development is made for the deficient LRs. All eleven sections are composed of single move configuration, which fall into theme conceptualization (Move 1). Particularly, knowledge presentation (1A) and surveying the research activities (1E) are the most frequently used submoves under theme conceptulization (Move 1).

There is no move use under the configuration types of "2-move configuration," "3-move configuration" and "4-move configuration." This tendency indicates that the writers just note down what they have read from the previous studies without any synthesis and indication of relevance. Whenever a new section starts, the topic is initiated at the knowledge presentation (1A, referring to introduction to theory, belief, practices or field knowledge) or surveying the research activities (1E, generally introducing the Introduction, Method, Results, Discussion of a study) or a combination of these two submoves (1A-1E). The LRs in the deficient group are found to have many topics standing alone in one section without presenting further discussion/comments carried out by moves $2 \mathrm{~A}$ and $2 \mathrm{~B}$ or providing proper link to the writers' proposed studies realized by the use of moves $3 \mathrm{~A}$ and $3 \mathrm{~B}$.

Also, the contents are to imitate the forms of the original works or studies in many aspects (such as organization, verbs, noun phrases, etcs). The knowledge presentation (1A) and activities surveying (1E) generally occupy great length with details in the LRs of this group. As a result, the topics tend to be out of focus if the lengthy texts are not carefully managed. Since the original works are not properly cited, the LR may be regarded as involving ethic problems, plagiarism, in academia. In the following, the researcher presents a typical text of the deficient group.

\subsubsection{Textual Analysis of the Low-rated Group}

As mentioned, the deficient writers use only the submoves under Move 1 and the sections are mainly composed of single move configurations. Partial texts in Section 4 of LR 28 are shown in Excerpt 1, in which the author presents approaches and objectives of culture instructions. Since this section covers eight pages, some texts are omitted. Only critical sentences are retained for discussion. In the selected LR texts, the number in the upper right-hand side at the beginning of each sentence is for readers to recognize the order of the sentence. For example, "1" in " $\mathrm{Li}$ (1994) compiled a great many approaches of teaching culture in the language classroom brought up and developed by various scholars or practitioners related to this field.... "stands for S1, meaning the first sentence of this passage. 
Excerpt 1. Selected LR Text in the Low-rated group

\subsubsection{Approaches and Objectives of Culture Instruction}

${ }^{1} \mathrm{Li}$ (1994) compiled a great many approaches of teaching culture in the language classroom brought up and developed by various scholars or practitioners related to this field..... ${ }^{2}$ According to $\mathrm{Li}(1994)$ the approaches share three common functions: cultural misunderstanding", "to engage in cultural encounters" and "to reinforce language learning" (p. 190). ${ }^{3}$ The seventeen approaches included by Li (1994) are the use of: (1) cartoon and cultoon,... (17) special programs. ${ }^{4}$ The following are the descriptions of the seventeen approaches along with their objectives:

${ }^{5}$ (1) cartoon and cultoon: The technique is used to teach not only the language components but also culture. It is difficult to clarify subtractions such as values, part of the covert culture, with written description. Chen (2003) advocated that.... This activity fits into conversation class for students to take part in discussion orally... (2) - (16) are skipped

\section{$1 \mathrm{~A}$}

(knowledge

presentation)

${ }^{6}(17)$ Special programs: In addition to the approaches employed in class, extra-curricular activities facilitate culture teaching if extra time, efforts and budgets are allowed....As to the use of the language festivals, the teacher can have the students to simulate fun activities in the target language and culture such as garage sale.

${ }^{7}$ Moran (2001) proposed a quite detailed and specific framework for learning/teaching culture.... ${ }^{8}$ The simplified version of Moran's (2001) summaries of the four knowings adapted by the researcher are as follow:

(1) Knowing How

- $\quad$ Content: skills

- $\quad$ Learning objectives: to demonstrate an ability

- $\quad$ Activities: dialogs, role plays, simulations, and field experiences

- $\quad$ Note: Communicative competence in the language and culture occurs. Students know both what to say and how to do it in a culturally appropriate manner.

- $\quad$ Teacher role: coach or model,... (2)-(3) are skipped

(4) Knowing Oneself

- Content: self-awareness

- Learning objectives: to demonstrate understanding of one's feelings, values, opinions, attitudes, and act upon them by behavior/statements.

- Activities: learners examine and make statements about themselves, reflective writing, and feedback on above activities.

- Note: Learners themselves are the subject matter in a process of guided self-discovery as they study their own values and their reactions to those of the culture. They decide whether or not to change.

- Teacher role: counselor or guide.

A description of domain knowledge is given in the form of listing in the single move configuration of the deficient group. As evidenced in Excerpt 1, the information about the seventeen approaches is repeated twice: the lead-in list is given in S3 and then the content for each is sequentially explicated. The list of the seventeen approaches has caused dullness and boredom in the six-page long explanation. The texts, within which no classification, integration and critiquing are found, has led to tediousness and has failed to link with other texts.

After demonstrating the seventeen approaches, the writer takes two pages to present Moran's four objectives starting from S7, following the same presentation pattern of Li's seventeen approaches. Readers may get confused because the relationship between the seventeen approaches and the four objectives are not established in any sentences either 
in the topical sentence or the transitional sentence. No clue can be found to connect the review of these two topics in the excerpt. In the low-rated LRs, most of the LR texts are found to display background knowledge presentation without a synthetic review and a critical commentary required (Lather, 1999; Strike and Posner, 1983).

Also, in this LR text, "author prominent citations" are identified. "Author prominent citation" is defined as that the author rather than the information is regarded as more important in the information status (Weisseberg \& Buker, 1990). That is, "author" is taken as the topic. In Excerpt 1, the two authors (See S1 and S7), Li and Moran, serve as the subjects of the paragraphs and are placed in the beginning of each paragraph in the case of LR 28. Right after the subjects of the sentences, the claimed knowledge in this LR is stated: the seventeen techniques and the four objectives for culture instruction.

By using author prominent citation, the deficient writers faithfully include the contents from the original works, which testify that the author is not able to make textual link with other texts and to make a synthesis based on the theme or organize a key concept from these contents. For example, as the two topics "approaches" and "objectives" of culture instruction are put together, two paragraphs are assigned to the two cited authors' statements, which together is marked as "field knowledge presentation (1A)"; no relation is established to explain why the two topics are discussed together in this section and no further analysis and comments are made.

To conclude for the deficient group, the lack of topic development in reviewing the studies is the major weakness of their LRs. The topic selected restricts itself to the theme conceptualization throughout each section in which "knowledge presentation (1A)" and "surveying research activities (1E)" are extensively used. Idea listing and author prominent citations are found to be the textual features of this group. In the two LRs of this group, no submoves of Moves 2, 3, and 4 are used for discussion, critical comments, relevancy claiming and announcement of the proposed studies.

\subsubsection{The High-rated Group}

In the high-rated group, there is usually a complete cycle found in an LR; however, it is more frequent to find cycles in an LR for the distinctive writers. While the competent writers use more complicated move configuration, the distinctive writers are conscious of using multiple move cycles to achieve their communicative purposes in the LRs. In a word, the high-rated group displays the following text features: more cycles are completed in each LR of this group; in addition, multiple move configurations are used to elaborate on discussions. Table 7 shows the move use by the distinctive writers. There are five distinctive LRs in this study.

Table 7. Sectional Move Use by the Distinctive Group

\begin{tabular}{|c|c|c|c|c|c|c|}
\hline $\begin{array}{l}\text { LR } \\
\text { No. }\end{array}$ & 1 & 2 & 3 & 4 & 5 & 6 \\
\hline \multicolumn{7}{|c|}{ Move $1,2,3,4$} \\
\hline 1 & $1 \mathrm{D}$ & $1 \mathrm{D}$ & 1A_1D_1A & 2A_2A_2 & 1A_2A_2B_2A & \\
\hline 1 & 1D & 1A_3A & $1 \mathrm{~A} \_3 \mathrm{~A}$ & $1 \mathrm{~A}-2 \mathrm{~A} \_3$ & 1A_1E_3B_4A & 1A_3A_2A_1D \\
\hline 2 & $1 \mathrm{D}$ & 1A_3B_1A_1E_4B_1E_3B & 1D_2A_1E_2 & $2 \mathrm{~A} \_3 \mathrm{~A}$ & 3B_4A & \\
\hline 2 & $1 \mathrm{D}$ & 1A_1E_4B & $2 \mathrm{~A} \_3 \mathrm{~B}$ & $2 \mathrm{~A}$ & $2 \mathrm{~A} \_3 \mathrm{~B} \_4 \mathrm{C}$ & 1A_2A_4B_4A \\
\hline 2 & $1 \mathrm{D}$ & $1 \mathrm{~A}$ & 1D_2A_2B_3 & $2 \mathrm{~A}$ & 2A_2A_3B_4A & 2A_1A_1A_2B \\
\hline
\end{tabular}

Table 7 reveals several features of the move use by the high-rated group. First, more move cycles are completed in most of the LRs. The move structures are found to have two types in this group (see Table 7): an LR with one complete cycle (see LR 1) and LRs with more cycles (see LR 19, 20, 21, 27). There are multiple cycles because there are smaller cycles completed for subtopics which can be identified in certain section(s) in addition to a larger cycle (global cycle) completed at the end of the LR chapter. From this perspective, four LRs (LR 19, LR 20, LR 21 and LR 27) have multiple cycles. In this way, there are 15 cycles found in this group (see the shaded area in Table 7): one complete cycle is found in LR 1, 3 cycles in LR 19, 5 cycles for LR 20, 3 cycles for LR 21 and 3 cycles for LR 27. The average amounts to 3 cycles per LR in the group.

Second, the distinctive writers use multiple configurations to complete the cycles. It is found that cycles are completed within a section or across sections in most of the LRs in this group. For example, the researcher finds that LR 19 incorporates three cycles. LR 19 includes three topics in each configuration: "1A_2A_3B_4A" (section topic: 
Review of ESP Studies) for the $3^{\text {rd }}$ section, "1A_1E_3B_4A" (section topic: Review of Cohesion Studies) for the $4^{\text {th }}$ section and "1A_3A_2A_1D_1A_3B_4A" (section topic: Review of Reference Studies) for the $5^{\text {th }}$ section. The topic in each section is explored by using some combinations of the submoves under Moves 1, 2, 3 and 4. Discussions are made through cycles for the themes in that synthesis (2A), critiquing (2B), relevancy claiming (3A), gap indicating (3B) are frequently used to clarify the problems encountered in a field and to present the arguments. Compared with the extensive use of field knowledge presentation $(1 \mathrm{~A})$ in the other groups, the high-rated group reveals less reliance on knowledge presentation (1A). Most of the knowledge presentation (1A) presented in the configurations is immediately followed by submoves under Move 2 and Move 3. Knowledge presentation (1A) seldom stands alone. This shows that the knowledge presentation does not stop at Move 1. Instead, the discussion on field knowledge is further developed to discuss the pitfalls of past studies (2B) and consequently lead to indication of gaps among literature (3B).

The researcher also examines how the configurations are made up in this group. The findings in Table 8 show that the use of 3-move configurations and 4-move configurations are increasing than that in other groups. Table 8 shows the frequency of move configurations classified by single move, 2-move, 3-move and 4-move configurations. These classifications are used for the analysis of the move structure within configurations.

Table 8. Frequency of Move Configurations in Sections or Subsections in Distinctive Group

\begin{tabular}{ll}
\hline Move Configuration & Frequency \\
\hline Single move configuration & \\
1D & 1 \\
1A & 1 \\
2A & 2 \\
1A_1D_1A & 1 \\
2A_2A_2B & 1 \\
Subtotal & 6 \\
\hline 2-move configuration & \\
1A_1E_4B & 1 \\
1A_3A & 2 \\
2A_3A & 1 \\
2A_3B & 1 \\
3B_4A & 1 \\
Subtotal & 6 \\
\hline 3-move configuration & \\
1A_1E_3B_4A & 1 \\
1D_2A_1E_2B_4A & 1 \\
1A_2A_4B_4A & 1 \\
2A_3B_4C & 1 \\
2A_2A_3B_4C & 1 \\
2A_1A_1A_2B_4A_4D & 1 \\
1A_3B_1A_1E_4B_1E_3B_1A_3B_4A_3A_4A & 1 \\
Subtotal & 7 \\
\hline 4-move configuration & 1 \\
1A_2A_3B_4A & 1 \\
1D_2A_2B_3B_4A & 1 \\
1A_2A_2B_2A_3B_4A & 23 \\
1A_3A_2A_1D_1A_3B_4A & \\
Subtotal & \\
\hline Total & 1 \\
\hline & \\
\hline & \\
A & 1 \\
\hline
\end{tabular}


First of all, there are few repetitions in the move configurations among the writers. Fewer repetitions indicate that every distinctive writer has their own discoursal plan to arrange their move structures, which are unique and unlikely to be the same with others. In Table 8, all the six LRs show entirely different topic developments. To further examine the frequency the move configurations, the researcher finds that most of the configurations appear only once, except that " $2 \mathrm{~A}$ " appears twice in single move configuration and " $1 \mathrm{~A}-3 \mathrm{~A}$ " twice in 2-move configuration. The variations in move configurations show that the arguments made for the initiated topics are specifically managed in order to address to research problems raised in the LRs. Therefore, these configurations are not identical among writers.

Next, the distinctive writers consciously direct the LR discourse towards Move 4 to make topic development. As shown in the move arrangement by the distinctive writers, most of the configurations have move development shown in Table 8. For example, in 2-move configuration, the first two configurations show that the first two initial moves "knowledge presentation (1A)" respectively advance to "announcing research method (4B)" and " relevancy claiming to the current study (3A)." In the same manner, the next two configurations reveal that the initial moves (2A) respectively progress to "relevancy claiming to the current study (3A)" and "gap indication (3B)" while the other configuration shows that the initial move "gap indication (3B)" goes forward to "announcing to the current study (4A)." All the configurations have topic developed instead of remaining at the same stage like those in the type of single move configuration. Likewise, in the types of 3-move configuration and 4-move configuration, all the LRs reach the final move to indicate a niche (4A and $4 \mathrm{~B})$. All the topic development shown in the configurations, except those placed in the very beginning, is directed to Move 4 in order to form cycles.

\subsubsection{Textual Analysis of the High-rated Group}

In this section, the analysis of the LR text in this group is presented. Since the two text features have been indicated in the previous section, the researcher selects a section in LR 1 (see Excerpt 2) as a textual representation for the high-rated group to show the multiple configurations and how cycles are completed in this configuration. Next, partial texts of L1 in this section are selected for textual presentation. In this example, "L1 interference" and "universal factor" are two theoretical explanations for the learners' difficulties in learning English relativization. Section 2.2 reviews one of the theoretical camps "Universal Factor," in which the results of the empirical studies based on the three theoretical claims, PFH, PDH and NPAH, are compared and contrasted.

Excerpt 2. LR Text in the High-rated Group

\subsection{Universal Factors}

${ }^{1}$ With all empirical support for L1 interference from studies on English RC acquisition, there has as well been counterevidence yielded from researchers working in the field. ${ }^{2}$ For instance, Liu (1998), in an inquiry into English RC acquisition by Taiwanese junior high students, found the influence of L1 to be minimal on her subjects' error patterns...

${ }^{3}$ Furthermore, on the issue of avoidance, or underproduction, of RCs, Bertkau (1976) explains the same phenomenon as reflecting not L1 interference but rather a common learning strategy on the part of L2 learners to simplify the target language by substituting simple constructions for complex ones (using independent clauses in place of complex RCs)... ${ }^{4}$ Given these arguments against the significance of L1 interference, there may be universal factors, as well as language-specific ones, at work in determining $\mathrm{RC}$ acquisition by ESL/EFL learners.

\subsubsection{Three Universal Hypotheses for English Relativization Difficulty}

${ }^{5}$ Different hypotheses have been proposed to account for the relative ease and difficulty of various RC types, regardless of L2 learners' native background.... ${ }^{6}$ They are the Parallel Function Hypothesis, the Perceptual Difficulty Hypotheses, and the Noun Phrase Accessibility Hierarchy Hypothesis, all based on different rationales and predicting different difficulty orders for English relativization.

${ }^{7}$ The Parallel function Hypothesis (PFH)...

${ }^{8}$ A second predictor hypothesis of English RC difficulty is Kuno's (1974) Perceptua Difficulty Hypothesis (PDH. )...

$1 \mathrm{~A}$

(knowledge presentation) 
${ }^{9}$ The last, and arguably the most influential, predictor hypothesis is put forward by Keenan and Comrie (1977).

\subsubsection{Previous Empirical Studies on the Three Universal Hypotheses}

$\ldots{ }^{10}$ Its focus is mainly on testing the validity of the three theoretical claims by investigating whether the accuracy order of ESL/EFL learners' performance of various RC types actually corresponds to the difficulty hierarchy as predicted by the three. ${ }^{11}$ Generally speaking, studies on the three predictor hypotheses, particularly the $\mathrm{PFH}$ and the $\mathrm{PDH}$, are characterized by mixed results.

$\ldots{ }^{12}$ Sheldon's PFH has been directly countered by evidence from L2 studies (e.g. Gass \& Ard, 1980; Flanigan, 1994). ${ }^{13}$ Flanigan (1994) found that his L2 child subjects...

${ }^{14}$ Likewise, Kuno's (1974) PDH has not found consistent empirical support in the SLA literature, albeit positive findings from, among others, Cook (1973), Ioup and Kruse (1977), Schumann (1980), Kubota (1993), and Izumi (2003)...

${ }^{15}$ As opposed to conflicting findings regarding the PFH and the PDH, L2 studies on Keenan and Comrie's (1977) NPAH have uniformly yielded positive results...

${ }^{16}$ As a valid predictor of the difficulty order of English relativization, the NPAH also serves as an alternative explanation for L2 learners' errors involving the use of resumptive pronouns and their behavior of avoidance, both of which have long been held as resulting from L1 interference. ${ }^{17}$ In view of the fact that pronoun copying (resumption) is found in L2 learners whose L1 does not employ nominal reflexes in RCs and even in native speakers of English, resumptive pronouns can be viewed as a universal initial strategy with which to reduce processing difficulty inherent in structural complexity (Pavesi, 1986). ${ }^{18}$ The extent to which resumptive pronouns are used is claimed to concur with the NPAH (Keenan \& Comrie, 1977; Gass, 1979, 1980; Flagnigna, 1994)... ${ }^{19}$ The psychological validity of the NPAH also manifests itself in accounting for L2 learners' underproduction of RCs, especially those lower on the accessibility hierarchy, e.g. prepositional object and genitive relatives (Gass, 1979, 1980; Gass \& Ard, 1984; Eckman et al, 1988; Yip, 1991).

${ }^{20}$ Strictly speaking, the disparities in empirical support for the three predictor hypotheses, as revealed in Table 4 , need to be interpreted with some caution, as they may have been a consequence of methodological differences. ${ }^{21}$ For one thing, studies on universal factors often vary in their research focus. ${ }^{22}$ Most of them do not investigate all the three hypotheses. ${ }^{23}$ Instead, often assuming that the support for one hypothesis would indirectly falsify the other two, they tend to examine only one particular hypothesis with little consideration for the others. ${ }^{24}$ For another, these studies differ to a great extent in their participatns' L2 proficiency and their elicitation measures. ${ }^{25}$ Some included advanced learners or employed productive tasks (e.g. sentence-combining, free writing, speech production), whereas others, intermediate learners or receptive tasks (e.g. comprehension questions, interpretation tasks, grammaticality judgments). ${ }^{26}$ It can be argued that different proficiency levels and testing instruments may yield different results concerning the validity of a particular hypothesis.

\subsubsection{Summary}

${ }^{27}$ This section has so far explored two potential sources of difficulties encountered by ESL/EFL learners in acquiring RCs: L1 interference and universal factors. ${ }^{28} \mathrm{On}$ the on hand, some researchers, especially those following the paradigm of the contrastive analysis (e.g. Schachter, 1974; Schachter et al., 1976; Bley...) have pointed out L1 interference as a significant or even the sole cause of RC error patterns and underproduction (either conscious avoidance or subconscious underuse) by L2 learners.... ${ }^{29}$ As an alternative to L1 transfer, others (e.g. Cook, 
1973; Ioup \& Kruse, 1977...) have approached the same issue from the point of view of universal factors.... ${ }^{30}$ Underlying these processing problems are three major hypotheses-Sheldon's (1974) Parallel function Hypothesis, Kuno's (1974) Perceptual Difficulty Hypothesis, and Keenan and Comrie's (1977) Noun Phrase Accessibility Hierarchy Hypothesis__... ${ }^{31}$ These mixed results may in effect be a direct consequence of differences in research methodology.

$\ldots{ }^{32}$ Furthermore, all these studies are limited in scope to the case of restrictive RCs. ${ }^{33}$ The issue of how L2 learners acquire (or use) non-restrictive RCs is rarely touched upon.

$\ldots{ }^{34}$ what seems to be still lacking is further research (1) which inquires into L2 learners' acquisition of NRRCs, the marked type of RCs in English; and (2) which probes into the functional aspects of RC acquisition, namely, the issue of how effectively L2 learners are able to employ English RCs for such pragmatic/discourse functions as identifying, characterizing, presenting, interpolating, and backgrounding...

[LR 1]

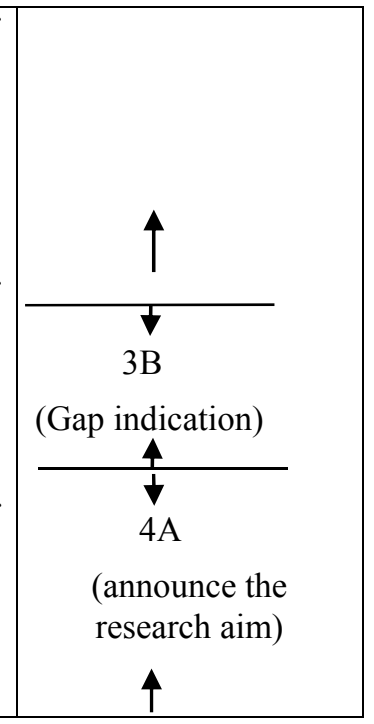

A cycle is completed in this section and the arguments are solidly grounded in the theories. The move structure comprises Moves 1, 2, 3 and 4, in which the submoves "knowledge presentation (1A)_synthesis (2A)_critiquing the research qualities (2B)_gap indication (3B)_announcing the aim of the current study (4A)" are used. As seen in the right column of the analysis of the LR text, the move structure comprises the submoves under Moves 1, 2, 3 and 4 . One or several functions of submoves contribute to each subsection, which constitutes a coherent move configuration and effectively forms a cycle in the three subsections of this configuration (Section 2.2.1-Section 2.2.3).

The arguments are solidly grounded in theories, realizing in Move 2 and Move 3. To begin with, under the heading 2.2 "universal factor," the author brings up several counter evidence for the explanations from L1 interference on relativization. Next, under the heading 2.2.1, several paragraphs are used to introduce the three universal hypotheses (see S5- S9). This subsection accounts for the submove use "presenting the field knowledge (1A)" under Move 1. Because the arguments grounded on the hypothesis will be discussed later in the text so that this knowledge needs to be introduced for readers to be familiar with. Next, Subsection 2.2.2 presents the empirical studies on these three universal hypotheses. The results of the empirical studies are discussed following the order of PFH, PDH and NPAH to compare and contrast the findings of these studies (see S10-S19). Therefore, this move is marked as a submove under Move 2, "synthesis (2A)," because a new perspective on the research results will be derived after the comparison and contrast of the results. The reviews has shown that there are mixed results based on PFH, and PDH but only the findings on NPAH remain consistent. Examining the findings based on the three hypotheses, the author concludes that there is a higher validity of NPAH. The following linguistic signs assist to recognize the submove use for synthesis:

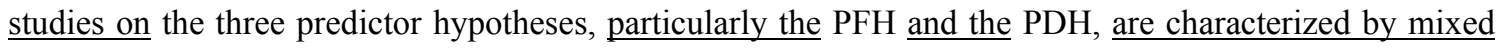
results $(\mathrm{S} 11)$.

Sheldon's PFH has been directly countered by evidence from L2 studies (e.g. Gass \& Ard, 1980; Flanigan, 1994) (S12).

As opposed to conflicting findings regarding the PFH and the PDH, studies on NPAH have uniformly yielded positive results (S15).

The underlined phrases such as "characterized by the mixed results," "countered by," "as opposed to" and "conflicting findings," "uniformly yielded positive results" are used to compare and contrast theories relating to the topic.

The last paragraph of the section focuses on the research qualities (2B), in which the methodological concerns are expressed (see S20-S26), including the participants' L2 proficiency and elicitation tasks. Also, the author cautions that the results on the three universal hypotheses should be interpreted carefully. In the summary subsection, the writer summarizes the two theoretical camps and synthesizes the research results (see S27-S31). The author uses the following phrases to represent his comments on the research qualities:

the disparities in empirical support for the three predictor hypotheses..., need to be interpreted with some 
cautions (S20).

(2) Studies on universal factors often vary in their research focus (S21).

Instead, often assuming that support for one hypothesis would indirectly falsify the other. They tend to examine only one particular hypothesis with little consideration for the other (S23).

These studies differ to a great extent in their participants L2 proficiency and their elicitation measures (S25).

It can be argued that different proficiency levels and testing instruments may yield different results concerning the validity of a particular hypothesis(S26).

The phrases that help reader to identify critiquing are usually accompanied by expression of justifications or suggestions. Therefore, "need to be interpreted with some cautions," "vary in their research focus," "differ to a great extent in," "It can be argued that..." are the phrases the author employs to offer explanations following the criticism made for the selected studies.

In addition to the linguistic signs found in phrases, the textual evidences are filled with information prominent citations (Weissburg \& Burg, 1990 p. 43). Information prominent citation is defined as the information given the primary focus rather than the author. The textual characteristic is that information is followed by a parenthesis in which there are the authors' names and year of publication. The authors indicated in the parenthesis can be referred to for the statements made. For example, in S18, S19, S28, S29, when the author summarizes a viewpoint, the citations are immediately attached for readers' references. As a textual characteristic, information prominent citation is used by the author to classify the same results from different empirical studies such as S18 and S19; it is used as a summary of the theoretical stance supported by more than one study (S28 and S29.) To conclude, information prominent citation as a textual characteristic is used to summarize the contents of the source articles in authors' own words with the aim to gather the viewpoints shared by the source articles in the parenthesis.

The high-rated writer successfully makes the move development from Move 1 to Move 4 and thus forms a cycle, in which the theoretical stance of the author's proposed study is justified. In the arguments, the writer negotiates with the existing knowledge (the universal hypothesis) instead of accepting them without challenging and examining the appropriateness of the theory(ies). The assessment of the findings is made plausible because the discussions are substantially based on the theories.

To conclude, multiple cycles are found in most of the LRs in this group. More cycles are completed because the distinctive writers make elaborated discussions on respective topics raised in the LRs. Three-move configurations and 4-move configurations are the dominant configurations types, in which the topic discussions are unanimously led to reach final move-announcing their focal studies. Also, information prominent citation is the key text feature to summarize the contents from multiple source articles and analyze the viewpoints shared from them.

\section{Conclusion}

In response to the three research questions, through move configurations, the literature review quality of each level could be analyzed. Move configurations coded for the literature reviews show the text constructions that different level of LR writers compose. In addition to the number of move uses increased from one move to four major moves as the level advanced, we also find that a move cycle is usually a must in writing up a literature review discourse. Higher-level moves (Move 3 and 4) are used more frequently by the higher-rated writers than by low-rated writers. Low-rated LRs focus mainly on received knowledge presentation in a certain field.

Past studies have shown that genre knowledge plays a crucial role in guiding students' EAP writing. According to the results, the literature reviews of low-rated writers lacked genre knowledge (Berkenkotter \& Huckin, 1995; Bitchener \& Basturkmen, 2006; Cheng, 2007; Dong, 1998; Hills et al., 1982; Swales \& Feak, 2000). Literature reviews written by the focal postgraduates were simply a sequential summary of articles, a list without plausible linkage and chronological structure (Boote \& Baile, 2005; Froese, Gantz, \& Henry, 1998; Swales \& Feak, 2000); this is a major weakness in literature reviews and often involves plagiarism and author prominent citation are dominant in the literature review discourse. Also, we also have found that little knowledge of creating a research space over an extended discourse with thorough coverage and balance (lacking of Move 3 and Move 4) are the difficulties that low-rated writers encounters. (Cooley \& Lewkowicz, 1995; Dong 1998, Jenkins et al., 1993).

In the current study, we found that half of the collected data were low-rated literature reviews. Accordingly, we suggest that the LR move structure and move configurations should be explicitly taught to postgraduates who take 
the thesis writing classes. However, this does not imply a static and decontextualized pedagogy. Novice theses writers can be guided in synthesis and paraphrasing practice. Also, thesis instructors can encourage postgraduates to analyze the move configurations of high-rated literature reviews by using the four major moves and their submoves. Thus, students will gradually master literature review discourse skills.

\section{References}

Belcher, D. (1994). The apprenticeship approach to advanced academic literacy: graduate students and their mentors. English for Specific Purposes, 13, 23-24.

Berkenkotter, G., \& Huckin, T. (1995). Genre knowledge in disciplinary communication. Hillsdale, NJ: Lawrence Erlbaum.

Bhatia, V. K. (1993). Analyzing genre: language use in professional settings. London: Longman.

Bitchener J. \& Basturkmen H. (2006).Perceptions of the difficulties of postgraduate L2 thesis students writing the discussion section. Journal of English for Academic Purposes, 5, 4-19.

Bloom, B., Engelhart, M. D., Furst, F. J., Hill, W. H., \& Krathwohl, D. R. (1956). Taxonomy of educational objectives: cognitive domain. New York: McKay.

Boote, D. N., \& Beile, P. (2005). Scholars before researchers: on the centrality of the dissertation literature review in research preparation. Educational Researcher, 34(6), 3-15.

Brett.P. (1994). A genre analysis of the results section of sociology articles. English for Specific Purposes, 13(1), 47-59.

Bunton, D. (1999). The use of higher level metatext in Ph.D. theses. English for Specific Purposes, 18, 41-56.

Cheng A. (2007). Understanding learners and learning in ESP genre-based writing instruction. English for Specific Purposes, 25, 76-89.

Cooley, L., \& Lewkowicz, J. (1995). The writing needs of graduate students' at the University of Hong Kong: A project report. Hong Kong Papers in Linguistics and Language Teaching, 18, 121-123.

Crookes, G. (1986) Towards a validated analysis of scientific text structure. Applied Linguistics, 7, 57-70.

Dong Y. (1998). Non-native graduate students' thesis/dissertation writing in science: self-reports by students and their advisors from two U.S. institutions. English for Specific Purposes, 17(4), 369-390.

Dudley-Evans, T. (1986). Genre analysis: An investigation of the introduction and discussion sections of MSc dissertations. In M. Coulthard (Ed.), Talking about text (pp. 128-145). Birmingham: English Language Research, University of Birmingham.

Froese, A. D, Gantz, B. S., and Henry, A. L. (1998). Teaching students to write literature reviews: a meta-analytic model. Teaching of Psychology, 25(2), 102-105.

Granello, D. H. (2001). Promoting cognitive complexity in graduate written work: Using bloom's taxonomy as a pedagogical tool to improve literature reviews. Counselor Education and Supervision, 40(4), 292-307.

Halliday, M. (1994). An introduction to functional grammar. London: Edward Arnold.

Halliday, M., \& Hasan. R. (1989). Language, context and text: aspects of language in a social semiotic perspective. Oxford: Oxford University Press.

Hart, C. (1998). Doing a literature review. London: Sage publications Ltd.

Hewings M. (1993). The end! How to conclude a dissertation. In G M Blue (Ed.), Language, Learning and Success: studying through English, Developments in TEFL. Hemel Hempstead: Phoenix TEFL.

Hill. S., Soppelsa B., \& West G. (1982). Teaching ESL students to read and write experimental-research papers. TESOL Quarterly, 16(3), 333-347.

Hopkins, A., \& Dudley -Evans, T. (1988). A genre-based investigation of the discussion section in articles and dissertations. English for Specific Purposes, 7, 113-122.

Hsiao, C., \& Yu, H. (2012). Knowledge Presentation in Thesis Writing- Examining Move Use in Reviewing Literature. English Teaching and Learning, 36(3), 1-47.

Hyland, K. (2004). Graduates' gratitude: the generic structure of dissertation acknowledgements. English for Specific 
Purposes, 23, 303-324.

Jenkins. S., Jordan, M., \& Weiland, P. (1993). The role of writing in graduate engineering education: a survey of faculty beliefs and practices. English for Specific Purposes, 12, 51-67.

Kwan, B. (2006). The Schematic structure of literature reviews in doctoral theses of applied linguistics. English for Specific Purposes, 25(1), 30-55.

Kwan, B. (2008). The nexus of reading, writing and researching in the doctoral undertaking of humanities and social sciences: implications for literature reviewing, English for Specific Purposes, 27, 42-56.

Lather, P. (1999). To be of use: the work of reviewing. Review of Educational Research, 69(1), 2-7.

Lau, H. (2003). The structure of academic journal abstracts written by Taiwanese PhD students. Taiwan Journal of TESOL. Taipei: National Chengchi University.

Lim, J. (2006). Method sections of management research articles: a pedagogically motivated qualitative study. English for Specific Purposes, 25(3), 282-309.

Liu, P. C. (2007). Learning how to write a good MA thesis literature review: a case study of one Taiwanese graduate student. Yuan Chih University, Unpubished master thesis.

Lores R. (2004). On RA abstracts: from rhetorical structure to thematic organization. TESOL Quarterly, 23, 280-302.

Mackey, A., \& Gass, S. M. (2005). Second language research: methodology and design. Mahwah, NJ: Lawrence Erlbaum Associates.

Martin, J. (1992). English text. Amsterdam: John Benjamins.

McNabb R. (2001). Making the gesture: graduate student submissions and the expectation of journal referees. Composition Studies, 29(1), 9-26.

Moravcsik M., \& Murugesan P. (1975). Some results on the function and quality of Citation. Social Studies of Science, 5, 86-92.

Nwogu, K. (1997). The medical research paper: structure and functions. English for Specific Purposes, 16(2), 119-138.

Paltridge B. (1994). Genre analysis and the identification of textual boundaries, Applied Linguistics, 15, 288-299.

Posteguillo, S. (1999). The semantic structure of computer science research articles. English for Specific Purposes, 18(2), 139-160.

Qian, J. (2003). Chinese graduate students' experiences with writing a literature review. MEd thesis, Queen's University at Kingston, Ontario, Canada.

Santos, M. (1996). The textual organization of research paper abstract in applied linguistics. Text, 16, 481-499.

Scott, W. (1955). Reliability of content analysis: the case of nominal scale coding. Public Opinion Quarterly, 17, 321-325.

Sim, J., \& Wright, C. (2005). The Kappa statistic in reliability studies: use, interpretation and sample size requirements. Physical Therapy, 85, 257-268.

Strike, K., \& Posner, G. (1983). Types of synthesis and their criteria. In S. Ward and L. Reed (Eds.), knowledge structure and use: implications for synthesis and interpretation (pp. 343-362). Philadelphia: Temple University Press.

Swales, J. (1981). Aspects of article introductions. Birmingham, UK: LSU, University of Aston.

Swales, J. M. (1990). Genre Analysis: English in academic and research settings. New York: Cambridge University Press.

Swales, J. M., \& Feak C. B. (2000). English in today's research world: A writing guide. Ann Arbor, MI: University of Michigan Press.

Weissberg, R., \& Buker, S. (1990). Writing up research: Experimental research report writing for students of English. NJ: Prentice Hall Regents.

Williams, P. (1999). Result sections of medical research articles; analysis of rhetorical categories for pedagogical purposes. English for Specific Purposes, 18, 347-366. 
Yang, R., \& Allison, D. (2003). Research articles in applied linguistics: moving from results to conclusions. English for Specific Purposes, 22, 365-385.

\section{Appendix}

Rating Scale of MA Literature Reviews

\begin{tabular}{|c|c|c|c|}
\hline 1 Deficient work & 2 Poor work & 3 Competent work & $4 \quad$ Distinctive work \\
\hline $\begin{array}{l}\text { No review of the } \\
\text { literature; give a listing } \\
\text { of what others have } \\
\text { written with no } \\
\text { integrated comments. } \\
\text { Unable to capture main } \\
\text { ideas in own words. } \\
\text { No arguments or key } \\
\text { variables identified } \\
\text { relevant to the topic. }\end{array}$ & $\begin{array}{l}\text { LR is simply sequential } \\
\text { annotations of some items } \\
\text { However, no arguments or } \\
\text { key variables identified } \\
\text { relevant to the topic. Able to } \\
\text { distinguish main ideas of the } \\
\text { articles reviewed. Though } \\
\text { the content will be based on } \\
\text { main ideas from source } \\
\text { articles, the paper is still } \\
\text { organized by source articles, } \\
\text { rather than topics or themes. } \\
\text { With each article reviewed, } \\
\text { there is a direct and explicit } \\
\text { link from the source article } \\
\text { to the current paper. }\end{array}$ & $\begin{array}{l}\text { Review of the main } \\
\text { literature with main } \\
\text { variables and arguments } \\
\text { identified. Some links } \\
\text { made to methodology } \\
\text { and justification. A slight } \\
\text { discussion of the results } \\
\text { from the source articles is } \\
\text { seen. Some attempts at } \\
\text { critical evaluation but the } \\
\text { attempt is weak in its } \\
\text { objective criteria. Each } \\
\text { article is directly and } \\
\text { explicitly linked to the } \\
\text { topic of the current paper. } \\
\text { However, the findings } \\
\text { from the source articles } \\
\text { are not linked to each } \\
\text { other. }\end{array}$ & $\begin{array}{l}\text { Comprehensive review of } \\
\text { the relevant literature; } \\
\text { systematically analyzed and } \\
\text { all main variables or } \\
\text { arguments identified. LRs } \\
\text { are thematically organized. } \\
\text { Each article is directly and } \\
\text { explicitly linked to the } \\
\text { theme of the current article. } \\
\text { Critical evaluation firmly } \\
\text { linked to justification and } \\
\text { methodology. Source articles } \\
\text { are analyzed and critiqued } \\
\text { based on strengths and } \\
\text { limitations. Both sides of an } \\
\text { argument are presented } \\
\text { without biased tones. } \\
\text { Conflict between or among } \\
\text { findings are presented. }\end{array}$ \\
\hline
\end{tabular}

Cited from Granello's (2001) Promoting Cognitive Complexity in Graduate Written Work: Using Bloom 's Taxonomy as a Pedagogical Tool to Improve Literature Reviews. Bloom, B., Engelhart, M.D., Furst, F.J., Hill, W.H., \& Krathwohl, D.R. (1956). Taxonomy of educational objectives: Cognitive domain . New York: McKay. 